\title{
The challenge of improving patient safety in primary care
}

Improving patient safety in primary care is critical for the NHS. The majority of clinical encounters occur in primary care, ${ }^{1,2}$ and the sector is becoming more exposed with earlier discharge from hospital, increasing prescriptions of potentially dangerous drugs by GPs, and an increasing fragmentation of services. ${ }^{3,4}$ Nonetheless, little research has been carried out into risk management in the primary care setting. ${ }^{5}$

Although primary care may in some ways constitute a less safe environment than acute care, it has a relatively small and flat organisational structure, and a strong tradition of multidisciplinary teamwork; both of which are important factors in fostering a safer culture. ${ }^{4}$

Nonetheless, some have maintained that there have historically been few effective levers for improving the quality and safety of primary care, partly due to GPs' status as self-employed contractors which has allegedly inhibited coordination and effective intervention. ${ }^{6}$ For some this was borne out by the relative lack of impact of the first Quality and Outcomes Framework (QOF) on the improvement of quality, given that so many practices were able to demonstrate performance above the stipulated thresholds. However, this may have been at least partly due to the fact that many were already achieving the targets. ${ }^{7}$

Two articles in this issue of the BJGP highlight a number of the challenges faced by those attempting to improve the safety of primary care. Pearson and colleagues $^{8}$ examine the role of GPs in relation to patients with mental health problems who have died from suicide, and Harnden et $a l^{9}$ consider the involvement of GPs in relation to childhood mortality.

Both studies suggest that there are actions which can be taken by GPs and others to ameliorate risks to patient safety in the primary care context. Hence, Harnden and colleagues shine a spotlight on failure to identify and act on signs of serious infections, meaning faulty diagnosis. Their analysis chimes with other studies which have underscored the significance of diagnostic error in primary care. ${ }^{1}$ Harnden and colleagues' study also highlights organisational factors that could lead to unsafe care, such as some GPs' failure to follow recommendations for vaccination by hospital doctors, and failure to follow-up non-attendance at appointments for those with conditions such as asthma and epilepsy.

Pearson and colleagues' study also draws attention to the challenges of effective coordination of care. For example, they suggest that GPs tend to rate the risk of suicide as being higher than do mental health teams. Such differences in risk assessment between professional groups is a consistent finding within the sociological literature on risk, ${ }^{10,11}$ but it is less clear how such professional differences may be overcome.

One way of improving inter-professional communication could come from the process of confidential inquiries, which both studies build on (concerning suicide ${ }^{8}$ and childhood deaths ${ }^{9}$ respectively). Such inquiries are generally well-received by practitioners from a variety of medical specialties, given their wide scope and involvement of well-respected experts.

Developing recommendations on the basis of such inquiries is a dangerous game, since it involves truncating the dependent variable (that is, the outcome seeking to be explained). Hence the inquiries, understandably, only focus on those using mental health services who did actually commit suicide rather than those whose attempts failed; on those children or pregnant women who did die rather than those who survived; and so forth.

This is a problem given the pervasiveness of 'hindsight bias'. ${ }^{12}$ The analysis of 'near misses' might help in this regard. Obviously, the level of incident reporting within primary care is extremely low: only one in 200 reports in the National Reporting and Learning System, operated by the National Patient Safety Agency, came from GPs last year. ${ }^{13,14}$ However, an alternative approach may be to ensure that 'near misses' are made subject to audit locally, as well as other adverse events in individual practices, following the inclusion of significant event audits within the revalidation process for GPs and the QOF. ${ }^{15}$ Some authors have suggested that GPs could be further incentivised to, for example, appoint personal doctors for very ill or dying patients and those with complex health problems, ${ }^{16,17}$ although the use of such incentives remains controversial.

Other actions could also be taken to improve patient safety which are not considered by the two articles mentioned above. The development of automated analysis of electronic sources of information (such as patient records, where available) to highlight unsafe care could help to identify potentially unsafe practices before incidents occur; this has not yet been extensively developed in the primary care context beyond electronic prescribing. ${ }^{18}$

Finally, it has been suggested that greater publicity could be given to comparative information concerning the quality of care between trusts and practices. This would, however, require strong measures to be taken to prevent 'gaming' and to contextualise any such data in relation to important factors, such as social class, ethnicity, and case load. ${ }^{19}$ One approach might be to highlight 'good practice' across the sector, although it should be remembered that it is often difficult to transfer such successful initiatives to other contexts in the absence of the appropriate cultural, social, and organisational conditions.

\section{Anneliese Dodds,}

Lecturer in Public Policy, King's Institute for the Study of Public Policy and Director, Risk 
Programme, NIHR King's Patient Safety and Service Quality Research Centre, King's College London.

\section{Naomi Fulop,}

Professor of Health and Health Policy and Director, NIHR King's Patient Safety and Service Quality Research Centre, King's College London.

\section{Provenance}

Commissioned; not peer reviewed.

\section{REFERENCES}

1. Kostopoulou O, Delaney BC, Munro CW. Diagnostic difficulty and error in primary care - a systematic review. Fam Pract 2008; 25(6): 400-413.

2. Kuzel AJ, Woolf SH, Gilchrist VJ, et al. Patient reports of preventable problems and harms in primary health care. Ann Fam Med 2004; 2(4): 333-340.

3. Lehmann LS, Puopolo AL, Shaykevich S, Brennan TA. latrogenic events resulting in intensive care admission: frequency, cause, and disclosure to patients and institutions. Am J Med 2005; 118(4): 409-413.

4. Wilson T, Pringle M, Sheikh A. Promoting patient safety in primary care. $B M J 2001 ; 323(7313)$ : 583-584.

5. O'Dowd A. Risks to patients in primary care need to be scrutinised. BMJ 2009; 338: b525.
6. The Shipman Enquiry. Fifth report - safeguarding patients: lessons from the past - proposals for the future. HMSO: London.

7. Graffy J, Griffin S. Review of the Quality and Outcomes Framework for diabetes: current indicators 2007-2008. Manchester: National Primary Care Research and Development Centre, 2008. www.npcrdc.ac.uk/Publications/Diabetes_200708.pdf accessed 13 Oct 2009).

8. Pearson A, Saini P, Da Cruz D, et al. Primary care contact prior to suicide in individuals with menta illness. Br J Gen Pract 2009; 59(568): 825-832.

9. Harnden A, Mayon-White R, Mant D, et al. Child deaths: confidential enquiry into the role and quality of UK primary care. Br J Gen Pract 2009; 59(568): 819-824.

10. Tulloch J, Lupton D. Risk and everyday life. London: Sage Publications, 2003.

11. Douglas M, Wildavsky AB. Risk and culture: an essay on the selection of technical and environmental dangers. Berkeley, CA: University of California Press, 1983.

12. Szmukler G. Homicide inquiries: what sense do they make? Psychiatr Bull R Coll Psychiatr 2000; 24(1): 6-10.

13. Hoffmann B, Beyer M, Rohe J, et al. 'Every error counts': a web-based incident reporting and learning system for general practice. Qual Saf Health Care 2008; 17(4): 307-312.

14. Day M. Primary care pays only 'lip service' to clinical governance, MPs say. BMJ 2007; 335(7619): 529.

15. Mashta O. Health check indicators will reflect local priorities. BMJ 2008; 336: 635.
16. Richards $\mathrm{T}$. Who is at the helm on patient journeys? BMJ 2007; 335(7610): 76 .

17. Roland M. Assessing the options available to Lord Darzi. BMJ 2008; 336(7645): 625-626.

18. Wilson T, Sheikh A. Enhancing public safety in primary care. BMJ 2002; 324(7337): 584-587.

19. Marshall MN, Hiscock J, Sibbald B. Attitudes to the public release of comparative information on the quality of general practice care: qualitative study. $B M J$ 2002; 325(7375): 1278

DOI: 10.3399/bjgp09X472845

\section{ADDRESS FOR CORRESPONDENCE}

\section{Anneliese Dodds}

King's Institute for the Study of Public

Policy, King's College London,

The Strand, London WC2R 2LS.

E-mail: anneliese.dodds@kcl.ac.uk 